\title{
ABDOMINOPLASTIA POR PLICATURA ANTERIOR LONGITUDINAL NA SÍNDROME DE PRUNE BELLY: EXPERIÊNCIA INICIAL DO HOSPITAL MUNICIPAL JESUS
}

\author{
ABDOMINOPLASTY BY LONGITUDINAL ANTERIOR PLICATURE IN PRUNE \\ BELLY SYNDROME: PRELIMINARY EXPERIENCE IN HOSPITAL MUNICIPAL \\ JESUS
}

\author{
Lisieux Eyer de Jesus, TCBC-RJ ${ }^{1}$ \\ Henrique Pessoa Ladvocat Cintra ${ }^{2}$ \\ Isabel Cristina Soito ${ }^{3}$ \\ José de Ribamar Valle ${ }^{3}$
}

\begin{abstract}
RESUMO: Objetivo: Apresentar os resultados iniciais com o uso da técnica de abdominoplastia longitudinal anterior por plicatura parietal descrita por Furness et al (1998) no Hospital Municipal Jesus. Método: Descrevemos as características e resultados dos primeiros pacientes tratados em nosso serviço, inclusive duas modificações adotadas com relação à técnica descrita, a saber, a exploração retroperitoneal sistemática para cura da criptorquia bilateral simultânea à abdominoplastia e a celiotomia "de segurança" para todos os pacientes operados. Resultados: O seguimento dos pacientes foi de 1,5 ano, 15 meses e 10 meses, respectivamente. Foi possível demonstrar que a técnica adotada foi de fácil execução e pequena morbidade, com resultados estéticos satisfatórios, grande melhora das condições psicológicas e do convívio social para os pacientes desde o pós-operatório imediato, melhora da constipação crônica, capacidade aeróbica e postura. Quanto às repercussões urodinâmicas, aguardamos reavaliação a longo prazo, para conclusões mais efetivas. Conclusão: A abdominoplastia longitudinal anterior é uma técnica de fácil execução e segura para o tratamento da síndrome de prune belly e melhora a capacidade funcional muscular abdominal. Tem conseqüências urodinâmicas, que necessitam de avaliação após longo prazo.
\end{abstract}

Descritores: Síndrome de Prune Belly; Abdominoplastia.

\section{INTRODUÇÃO}

A síndrome de prune belly (SPB) é doença incomum e sua expressão habitual é restrita ao sexo masculino (incidência populacional de até 1:35.000 nascidos vivos, 1,8\% do total de defeitos abdominais congênitos $\left.{ }^{1-3}\right)$. Caracteriza-se por criptorquia bilateral abdominal, dimorfismo da musculatura abdominal anterior, principalmente inferior e medial, e uropatia de grau variável, à qual se associa fre- qüentemente disfunção renal secundária. Normalmente estão associadas doenças do aparelho locomotor (principalmente pé torto congênito e doenças da coluna) e do aparelho respiratório, sejam elas morfológicas (hipoplasia pulmonar e deformidades torácicas do tipo (pectus excavatum ou carinatum), infecciosas (pneumonias de repetição) ou funcionais.

A preocupação médica com relação a estes pacientes está relacionada à preservação do aparelho urinário e da

1. Cirurgiã do Serviço de Emergência HUAP/UFF. Cirurgiã Pediátrica do Hospital Municipal Jesus/ SMS/RJ. Mestre em Cirurgia Abdominal da UFRJ.

2. Cirurgião Plástico do Hospital Municipal Jesus. Titular da Sociedade Brasileira Cirurgia Plástica.

3. R2 de Cirurgia Pediátrica do Hospital Municipal Jesus.

Recebido em 12/04/2000

Aceito para publicação em 20/02/2000

Trabalho realizado no Serviço de Cirurgia Pediátrica do Hospital Municipal Jesus (RJ). 
função renal, sendo a correção do defeito da parede abdominal relegada a um desconfortável segundo plano. Esta situação se agravava pela ausência de uma técnica eficaz e de baixa morbidade que permitisse tal correção, (embora tentativas neste sentido venham sendo elaboradas desde 1967 por Randolph $)^{4}$, e pelo desconhecimento quanto à importância fisiológica da parede abdominal normal, o que considerava as reconstruções abdominais na SPB como fundamentalmente estéticas e relativamente supérfluas para pacientes com problemas urológicos freqüentemente graves 5 .

Modernamente se reconhece a importância da mecânica da parede abdominal na função respiratória, intestinal e urodinâmica e há um novo enfoque quanto à necessidade e faixa etária preferencial para a reconstrução da parede abdominal nos portadores da síndrome, ao lado de novas técnicas de abdominoplastia ${ }^{6-8}$. A técnica descrita por Furness em 1998, que chamaremos aqui de abdominoplastia anterior longitudinal por plicatura da parede (AALPP) surgiu como nova alternativa com morbidade mínima para estes casos. Não envolve perda de qualquer segmento de parede abdominal, é de execução fácil e não prejudica reoperações abdominais, se necessário, através de incisão mediana. Relatamos aqui a experiência do HM Jesus com a AALPP em três pacientes, na que acreditamos ser a primeira casuística nacional com a técnica em questão.

\section{MÉTODO}

Metodologia cirúrgica (Figura 1): Paciente colocado em decúbito dorsal e posição de Trendelenburg. Marcação da pele sob tração controlada da área provável de ressecção cutânea (Figura 1-A). Incisão mediana infra-umbilical por planos e celiotomia. Dissecção dos testículos, deixando amplo retalho peritoneal em torno dos vasos testiculares e deferenciais. Ligadura proximal dos vasos gonadais ${ }^{9}$, orquidopexia bilateral através de neo-anel modelado através da parede abdominal. Incisão cutânea mediana xifopubiana com circundução completa da cicatriz umbilical, mantendo-a inserida na parede abdominal anterior (Figura 1-B). Dissecção no plano subcutâneo. liberando os retalhos cutâneos marcados inicialmente, da aponeurose subjacente até os flancos, ultrapassando a marcação inicial conforme necessário para a plicatura da aponeurose a ser feita a seguir (Figura 1-C). Confecção de duas pregas incluindo todo o plano mioaponeurótico da parede abdominal, entre a linha hemiclavicular média e a porção média do ligamento inguinal, estabilizando-as com pontos de fio inabsorvível, protegendo as vísceras abdominais (Figura 1-D). Fechamento da incisão infra-umbilical mediana. Síntese das pregas de parede entre si na linha média (Figura 1E). Ressecção da sobra de pele na linha média. Síntese cutânea e refixação da cicatriz umbilical (Figuras 1-F e 1G). Drenagem no plano do tecido celular subcutâneo através de drenos de aspiração e curativo compressivo.

O primeiro paciente operado no Hospital Municipal Jesus foi um menino de dois anos de idade (Figura 2-A), já submetido à orquidopexia direita por técnicas tradicionais (acesso inguinal e técnica de Fowler-Stephens) ${ }^{9}$, com atrofia testicular ipsilateral completa. Apresentava megabexiga, megaureter e hidronefrose direita de pequena intensidade, megaureter e hidronefrose esquerda graves, com grau moderado de atrofia cortical à esquerda. Foi realizado esvaziamento completo de ambos os sistemas coletores mediante injeção de furosemida com ausência de refluxo vesicoureteral. A urodinâmica era normal, exceto por resíduo pósmiccional significativo. No pré-operatório ocorriam, em média, três infecções urinárias por ano. A evacuação era em dias alternados, muito lenta e pouco volumosa. Apresentava crises de "bronquite" e "resfriados" freqüentes, tórax com leve deformidade em sino e diminuição do diâmetro anteroposterior. Foi operado em maio 1999. No pósoperatório não houve intercorrências graves e ocorreu infecção de parede verificada com 12 dias e tratada apenas com drenagem. O paciente permaneceu com cinta abdominal compressiva por três meses no pós-operatório. O testículo esquerdo era tópico e sem sinais de atrofia. Os resultados estéticos foram satisfatórios para a criança e familiares (Figura 2-B). A fraqueza de musculatura abdominal em flanco direito, verificada desde o peroperatório, evidenciou melhora progressiva. A capacidade motora melhorou e a atividade física passou a ser desempenhada com menos cansaço e mais vigor. Não apresentou qualquer disfunção ou infecção respiratória no pós-operatório, e houve melhora da função intestinal. Inicialmente a freqüência miccional estava aumentada, sem ocorrência de infecções urinárias. No momento a criança está com três anos de idade, continente para fezes e urina, freqüentando escola regular (seguimento de 1,5 ano).

O segundo caso operado foi de um paciente de 11 anos de idade, que após exploração inguinal há dois anos visando tratamento de criptorquia, com diagnóstico de "testículo direito ausente" apresentava megabexiga, hidronefrose moderada e dolicomegaureter bilateral. A função renal mantida bilateralmente simétrica e havia esvaziamento completo dos sistemas excretores com o uso de diurético (furosemida) e ausência de refluxo vesicoureteral. Não havia história conhecida de infecção urinária ou respiratória. Referia operação urológica com finalidade desconhecida com um ano de idade. Não tinha avaliação urodinâmica pré-operatória e havia relativa constipação intestinal leve. Havia deformidade torácica em sino com diminuição do diâmetro torácico anteroposterior e história de dispnéia aos médios esforços e incapacidade de praticar esportes ou brincadeiras que exijam esforço físico. Apresentava problemas sociopsíquicos graves, com rejeição materna declarada pela presença da deformidade e convívio precário com as demais crianças. Submetido à operação em outubro 1999, o pós-operatório imediato ocorreu sem qualquer anormalidade. Permaneceu com cinta abdominal compressiva por três meses no pós-operatórios. Os testículos eram tópicos e sem sinais de atrofia no pós-operatório. O resultado estético foi ótimo (Figura 3-A), deixando o paciente muito satisfeito. A situação sociofamiliar melhorou, como o convívio com outras crianças. Houve melhora da função intestinal, com acentuado aumento da capacidade aeróbica, executando esforços físicos moderados sem dificulda- 


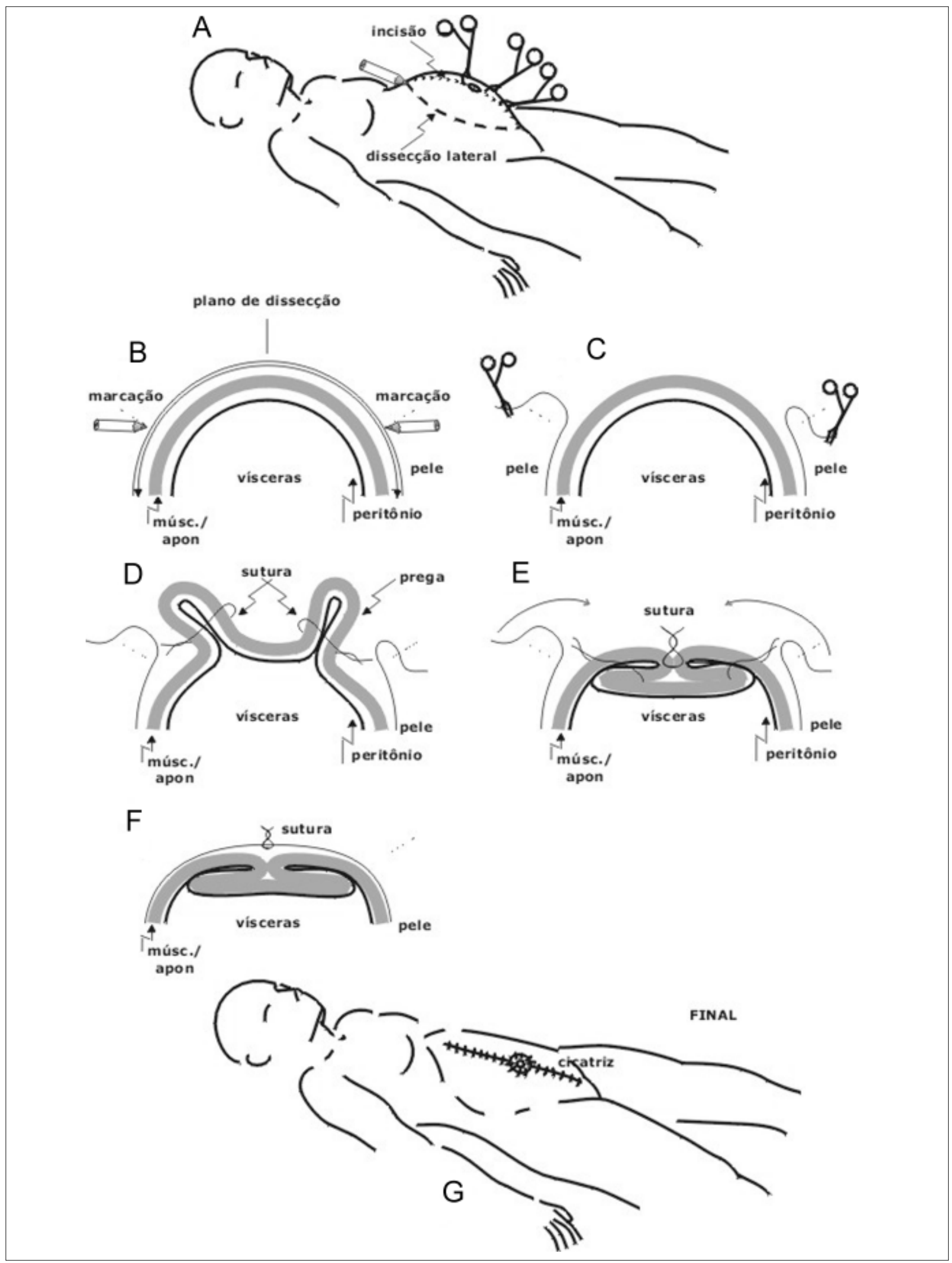

Figura 1 - Metodologia cirúrgica, descrição no texto. 


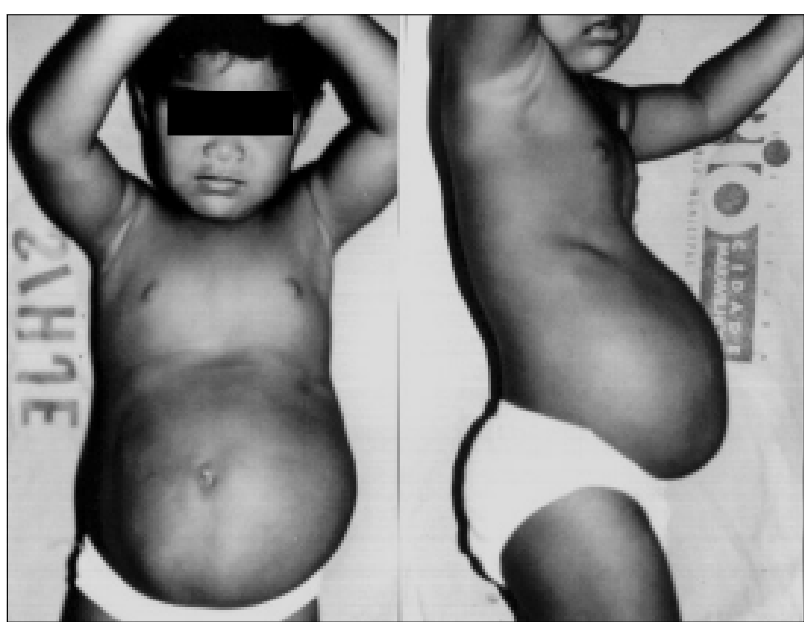

Figura 2A - Paciente 1, pré-operatório.

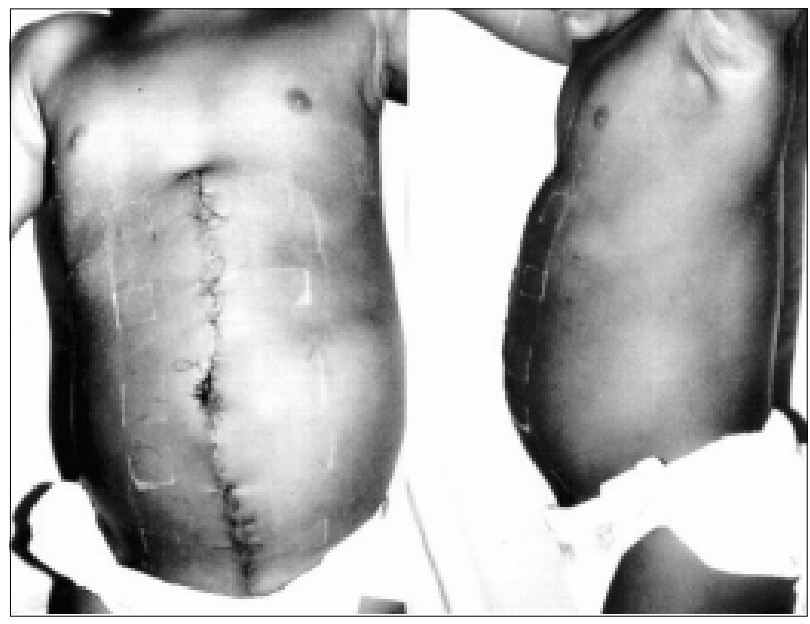

Figura 2B - Paciente 1, pós-operatório.

de (Figura 3-B). Apresentou polaciúria, com melhora espontânea lenta, sem incontinência e sem episódios de infecção urinária no período pós-operatório, mas referindo episódios eventuais de enurese noturna, com freqüência progressivamente melhor (seguimento de 15 meses).

O terceiro caso doente é de um adolescente de 16 anos de idade, tratado no Serviço de Cirurgia Pediátrica desde neonato, com diagnóstico de síndrome de prune belly. Submetido inicialmente a pielostomias bilaterais, revertidas aos quatro anos de idade, após reimplante ureteral bilateral. Foi submetido a orquidopexias direita e esquerda pela técnica de Fowler-Stephens ${ }^{9}$, realizadas respectivamente aos quatro e seis anos de idade, com bons resultados. Assintomático no momento, exceto por urinar com pouca freqüência (apenas três vezes ao dia), grande volume e jato "fraco". As provas de função renal são normais, com ausência de refluxo vesicoureteral e hidroureteronefrose bilateral moderada sem sinais obstrutivos. Relata dificuldade em suportar exercício físico moderado (por exemplo subir escadas), usa cinta de contenção para suportar

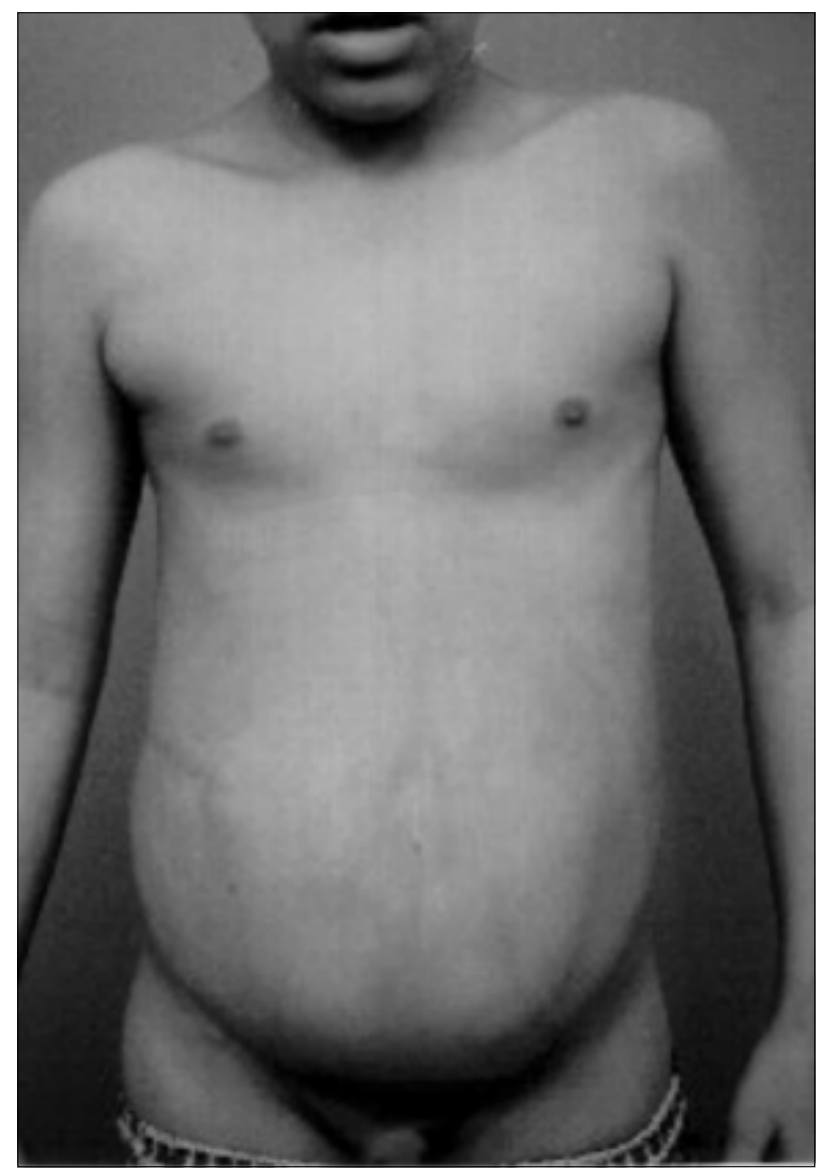

Figura 3A - Paciente 2, pré-operatório.

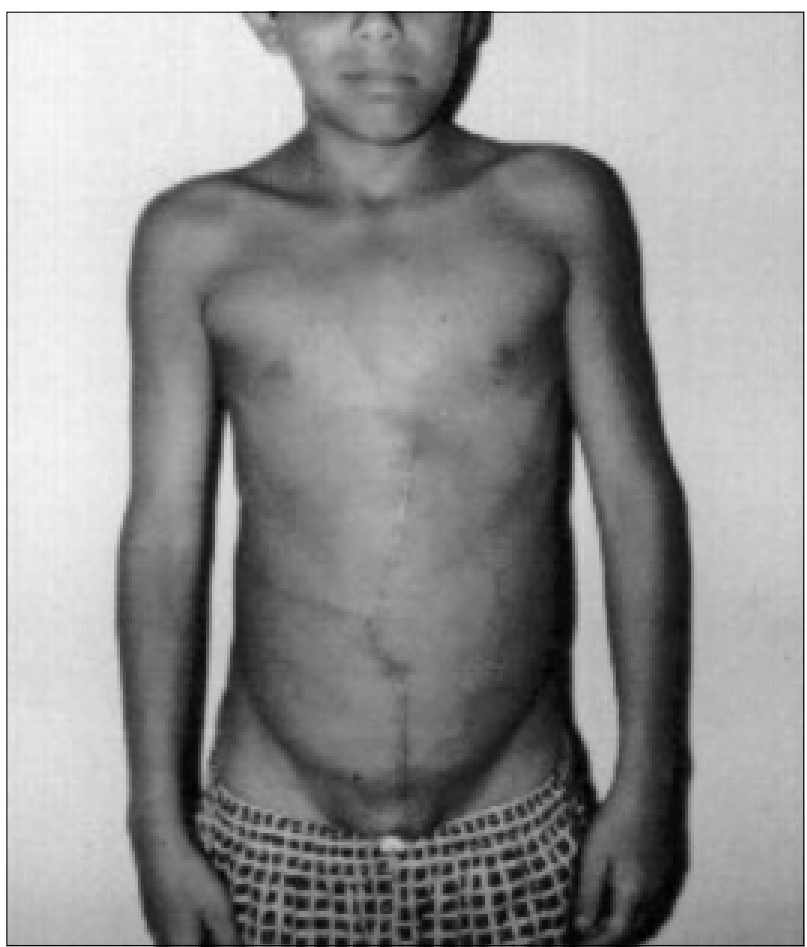

Figura 3B - Paciente 2, com dois meses de pós-operatório. 
esforços e manter aspecto "mais normal". Procurou espontaneamente o serviço ao saber da existência de uma chance de correção de sua deformidade, o que desejava ardentemente desde a idade escolar. Foi submetido à operação em abril de 2000. O pós-operatório ocorreu sem problemas, exceto pequeno seroma de ferida operatória. Permaneceu usando ininterruptamente a cinta abdominal durante três meses. $\mathrm{O}$ resultado estético foi excelente e o paciente ficou extremamente satisfeito alegando se sentir recompensado pelo tratamento. Refere melhora subjetiva em executar esforços físicos e do padrão de evacuação. Apresenta a polaciúria inicial, que melhorou espontaneamente após dois meses. Está aguardando exame urodinâmico e relata melhora da micção, com jato urinário mais potente (seguimento de 10 meses).

\section{DISCUSSÃO}

A abdominoplastia nos portadores de síndrome de prune belly foi descrita utilizando várias técnicas 4; 6-8. A primeira delas, descrita por Randolph em $1967^{4}$ se baseava na ressecção transversa extensa da parede, predominantemente em sua porção mais defeituosa, através de uma incisão abdominal inferior transversa "em sorriso" que se prolongava até os flancos em plano total. Os resultados pouco satisfatórios e principalmente pouco duradouros, com vários casos de recorrência, e a técnica cirúrgica relativamente agressiva não tornaram o método amplamente utilizado. Posteriormente outros autores idealizaram novas técnicas de abdominoplastia, em que a correção da parede se fazia com plásticas longitudinais da parede abdominal ${ }^{7-8}$, sem desinserir a parede abdominal inferior e sem ressecar segmentos parietais, criando dois retalhos laterais superpostos sobre a linha média (Ehrlich) ${ }^{6}$ ou retalhos medial ${ }^{1}$ e laterais ${ }^{2}$ que se superporiam na linha média (Monfort) ${ }^{7}$. Estas técnicas apresentaram resultados cosméticos e funcionais muito melhores, mas permaneceram exigindo operações extensas, com ampla exposição das vísceras intraperitoneais e grande potencial de complicações, especialmente sob forma de aderências e bridas. A técnica de AALPP, descrita em $1998^{8}$, propõe a solução destes defeitos potenciais das técnicas de correção abdominal no sentido longitudinal, descrevendo uma correção baseada em retalhos parietais obtidos pela plicatura de segmentos de parede, sem exigir ressecção ou incisão destes, originalmente abrindo mão até da abertura da cavidade abdominal para a execução da intervenção.

Em nossa casuística realizamos a técnica com pequenas modificações, levados pela convicção quanto às vantagens de executar as orquidopexias necessárias para estes pacientes através de uma exposição retroperitoneal ampla, que possibilite a criação de retalhos de peritônio posterior capazes de auxiliar na preservação da rede vascular colateral, através das artérias deferenciais após a laqueadura obrigatória das gonadais nos testículos intraabdominais e pela necessidade de garantir a segurança das vísceras abdominais quando da execução dos pontos de estabilização das pregas parietais, apesar desta necessidade ser negada no relato original. Indicamos a AALPP para pacientes após o primeiro ano de vida, estáveis quanto às condições renais e urológicas, em conjunto com a orquidopexia bilateral para a correção da criptorquia típica da síndrome, com celiotomia infra-umbilical. Temos indicado a operação inicialmente após a correção dos problemas urológicos, embora a princípio não haja contraindicação de executar a AALPP como etapa final complementar em uma intervenção urológica e não haja presumivelmente, dificuldades posteriores em executar alguma operação abdominal eventualmente necessária através de incisão mediana, sem lesar o reparo construído na AALPP.

A presença de doenças respiratórias não é uma contra-indicação para o tratamento cirúrgico, exceto situações agudas ou que impliquem risco anestésico proibitivo, já que evidências recentes indicam que os distúrbios respiratórios presentes na síndrome podem ser ao menos parcialmente revertidos pela presença de uma parede abdominal com capacidade de continência restaurada. A ausência de uma parede abdominal funcional provoca alterações graves da mecânica respiratória, com restrição séria da capacidade expiratória espontânea e forçada, dificuldade em exercer tosse efetiva para a eliminação de secreção, perda da força e amplitude do movimento do diafragma, em especial em ortostatismo. $\mathrm{O}$ único estudo quanto à função respiratória mecânica na SPB ${ }^{10}$ considera pacientes maiores que seis anos de idade, em sua maioria > 12 anos (por restrições técnicas à execução do exame), nenhum deles submetido a uma abdominoplastia considerada eficaz. É verificada diminuição importante na força e no fluxo expiratório, em especial em posição supina. Setenta e nove por cento dos casos apresentavam respiração paradoxal abdominal em repouso ortostático e $60 \%$ em exercício. Setenta e oito por cento dos pacientes tinham história pregressa de pneumonia, especialmente na primeira infância, e vários recusaram qualquer teste sob esforço por não suportarem exercícios físicos. Foi interessante notar que vários deles apresentaram relato espontâneo de melhora respiratória, especialmente se expostos a esforços, através do uso de cintas abdominais de contenção. O dado de que a doença respiratória secundária à SPB predomina na primeira infância é constante na literatura $^{5,10-12}$, mesmo após excluir os casos de hipoplasia pulmonar, o que se explica pela presença de uma menor força da musculatura respiratória auxiliar e uma parede torácica menos rígida, que em conjunto responderiam por uma menor capacidade de exercer toilette pulmonar e manobras respiratórias eficientes. Não foi possível a realização de provas de função respiratória pré e pós-operatória em nossos pacientes, mas todos referem grande melhora da capacidade de exercer esforços aeróbicos e o paciente em idade pré-escolar deixou de apresentar as complicações respiratórias verificadas antes da operação. 
A análise quanto ao comportamento urodinâmico é mais difícil, uma vez que é difícil separar as conseqüências das alterações pressóricas abdominais das conseqüências dos dimorfismos urológicos per se, especialmente aqueles devidos à típica megabexiga que ocorre nestes pacientes. Existem poucos estudos urodinâmicos dos pacientes portadores de SPB, e apenas um deles ${ }^{13}$ enfoca comparativamente pacientes antes e depois de abdominoplastia de Monfort. Neste estudo 90\% dos pacientes apresentavam dupla micção, $80 \%$ incontinência diurna e capacidade vesical grandemente aumentada (em média duas vezes a capacidade normal para a idade), com resíduo pós-miccional médio de $40 \%$ da capacidade vesical e aumento do limiar sensitivo para o desejo ou necessidade miccional. Após abdominoplastia foi verificada grande melhora nos índices, com resolução dos episódios de incontinência em $57 \%$ dos pacientes, diminuição do limiar sensitivo para a necessidade miccional e da capacidade vesical para 1,6 vez a normal. $\mathrm{O}$ volume residual médio caiu de $47 \%$ para $13 \%$ da capacidade vesical e a incidência de infecções urinárias de três a seis para um ano. Nossos pacientes aguardam ainda a avaliação urodinâmica formal, planejada para um ano pós-operatório, mas verificamos clinicamente um aumento da freqüência miccional desde o pós-operatório imediato, junto a um menor limiar quanto à necessidade miccional, produzindo polaciúria em todos os pacientes e episódios de incontinência no paciente pré-escolar. Embora aguardemos uma análise mais precisa de resultados é uma possibilidade a associação de cistoplastia redutora em casos futuros. Porém, as indicações precisas deste procedimento permanecem indistintas em literatura e há, reconhecidamente, a tendência para uma abordagem urológica minimamente invasiva para os portadores de SPB ${ }^{5}$.

A constipação intestinal nestes pacientes é devida à ausência da prensa abdominal funcional capaz de produzir manobra de Valsalva eficaz no momento da evacuação e pode ser grave, induzindo a megacólon adquirido de difícil controle, mesmo com colostomia. Temos verificado uma excelente resposta à AALPP quanto a este aspecto, também citados por outros autores, com todas as técnicas de abdominoplastia longitudinal, muito embora os relatos dependam de parâmetros clínicos de avaliação $0^{5,7,8,13}$. Os resultados da AALPP quanto aos problemas ortopédicos associados à SPB são apenas especulativos, supondo-se a melhora imediata de postura, provável melhora com relação aos distúrbios envolvendo a coluna vertebral (presumivelmente ligados a um desenvolvimento postural desequilibrado pela aplasia assimétrica do segmento muscular responsável pela postura troncular anterior) ${ }^{14}$ e tórax. Em nossos pacientes o tempo de seguimento ainda não permite conclusões definitivas a este respeito, embora já haja notadamente uma postura corporal melhorada, com redução espontânea da lordose verificada antes da operação.

Os aspectos sociais e psicológicos têm sido negligenciados nos pacientes de SPB, a nosso ver de forma errônea. Em nossa vivência clínica temos verificado uma grande projeção destes aspectos na qualidade de vida dos pacientes e suas famílias, seja na forma de complexos de rejeição e isolamento dos pacientes, seja pela impossibilidade de convívio social irrestrito imposto pela deformidade por si só - bastante relevante em nossa cultura e condições climáticas ou pela incapacidade de exercer atividades aeróbicas e esforços físicos significativos - relevante em escolares e adolescentes como instrumentos de integração no grupo escolar. Nosso paciente pré-adolescente nos disse no pré-operatório, ao ser avisado de que esta operação não era obrigatória, que se submeteria a qualquer procedimento, indiferente ao incômodo imediato que este pudesse causar-lhe, desde que houvesse uma chance de se tornar "mais parecido com as pessoas normais", "menos rejeitado" e pudesse "fazer xixi em pé", coisa que a deformidade abdominal lhe impossibilitava. A criança em idade préescolar não tinha queixas ou complexos de rejeição perceptíveis, mas se tornou mais sociável após a intervenção, e não perde qualquer oportunidade de exibir "a barriga nova e bonita" que agora apresenta. O paciente de 16 anos nos procurou espontaneamente ao ser informado de que havia uma chance de correção de sua deformidade, que lhe fora negada reiteradamente desde a infância, e referiu desejo de se submeter à técnica oferecida "imediatamente". Em nossa experiência há uma grande ansiedade quanto à possibilidade de correção da deformidade entre pacientes ambulatoriais ainda não expostos a qualquer forma de abdominoplastia, especialmente em pré-adolescentes e escolares, o que é confirmado pela totalidade dos autores envolvidos na correção de casos de SPB.

Quanto aos aspectos técnicos, julgamos a operação AALPP bastante simples e segura, associada facilmente à orquidopexia bilateral com exploração retroperitoneal. Julgamos de alto risco realizar o procedimento sem a exposição - mesmo mínima - da cavidade peritoneal, permitindo visualizar as vísceras intracavitárias ao serem colocados pontos de sutura para a confecção das pregas parietais laterais, ainda que esta manobra não seja aconselhada no relato original ${ }^{9}$ e presumivelmente aumente a morbidade do procedimento, podendo induzir aderências e bridas intestinais secundárias, que seriam ausentes quando não há exposição visceral. A dor pósoperatória nas primeiras 48 horas é problema sério pela intensidade. Temos optado pela inserção de cateter peridural e analgesia com opiáceos para controle, com sucesso. Os drenos de aspiração a vácuo foram retirados com 48 horas de pós-operatório. Os resultados estéticos foram satisfatórios, embora a cicatriz umbilical permaneça mais cranial que sua localização habitual. Ainda assim, a preservação da cicatriz umbilical original do paciente, sem a confecção de neo-umbigo, é considerada uma vantagem da técnica. Não houve em qualquer dos casos evidência de disfunção respiratória secundária à compressão diafragmática ou necessidade de ventilação assistida no pós-operatório. 


\begin{abstract}
S
Background: Our aim is tis present initial results with the anterior longitudinal abdominoplasty, described by Furness et al (1998) in three patients operated at the Hospital Municipal Jesus, Rio de Janeiro, Brazil. Methods: We describe pre and post-operative clinical data and surgical results for the first three patients submitted to surgical treatment and describe two technical modifications: retroperitoneal systematic exploration simultaneously to parietal abdominoplasty to treat cryptochid testicles and "for safety" celiotomy to protect abdominal viscera. Results: Follow up 1,5 year, 15 and 10 months for patients 1, 2 and 3, respectivelly. We could demonstrate that anterior longitudinal abdominoplasty is a safe and easily performed surgical technique, with good aesthetic results and significant psychological and social improvement. Chronic constipation, aerobic capacity and postural changes are also ameliorated. Regarding urodynamic repercussions we think that a longer period of observation is necessary to reach any definitive conclusions. Conclusions: Anterior longitudinal abdominoplasty is an effective and safe technique to treat prune belly syndrome patients and ameliorates functions of the abdominal parietes. It has urodynamic consequences that need long-term follow-up.
\end{abstract}

Key Words: Prune belly syndrome; Abdominoplasty.

\section{REFERÊNCIAS}

1. Loder, R; Guiboux, J; Bloom, DA et al. Musculosqueletal aspects of prune belly syndrome. Am J Dis Child 1992; 146:1224.

2. Druschel, CM. A descriptive study of prune belly in New York State, 1983-1989. Arch Pediatr Adolesc med 1995; 149:70.

3. Tan, Kh; Kilby, MD; Whittle, MJ et al. Congenital anterior abdominal wall defects in England and Wales 198793: retrospective analysis of OPCS data. BMJ 1996; 313:903.

4. Randolph, JG. Total surgical reconstruction for patients with abdominal muscular deficiency (prune belly) syndrome. J Pediatr Surg 1977; 12:1033.

5. Woodard, JR. Lessons learned in 3 decades of managing the prune belly syndrome - editorial. J Urol 1998; 159:1960.

6. Ehrlich, RW; Lesavoy, MA, Fine, RN. Total abdominal wall reconstruction in the prune belly syndrome. J Urol 1986; $136: 282$

7. Monfort, G; Guys, JM; Bocciardi, A et al. A novel technique for the reconstruction for patients with abdominal wall in the prune belly syndrome. J Urol 1991, 146:639.

8. Furness, PD; Cheng, EY, Franco, I et al. Prune belly syndrome: a new and simplified technique of abdominal wall reconstruction. J Urol 1998; 160:1195.

9. Fowler, R; Stephens, FD. The role of testicular vascular anatomy in the salvage of high undescended testis. Aust New Zeal J Surg 1959, 29:92.

10. Ewig, JM; Griscom, NT; Wohl, MEB. The effect of the absence of the abdominal muscles on pulmonary function and exercise. Am J Respir Crit Care Med 1996, 153:1314.

11. Brinker, MR; Palutsis, RS; Sarwark, JR et al. The orthopedic manifestations of prune belly (Eagle Barrett) syndrome. J Bone Joint Surg 1995; 77A(2):251.
12. Geary, DF, McLusky, IB, Churchill, BM et al. A broader spectrum of abnormalities in the prune belly syndrome. J Urol 1983; 135:324.

13. Smith, CA; Smith, EA; Parrott, TS et al. Voiding function in patients with the prune belly syndrome after Monfort abdominoplasty. J Urol 1998; 159:1675.

14. Loder, R; Guiboux, J; Bloom, DA et al. Musculoskeletal aspects of prune belly syndrome. Am J Dis Child 1992, $146: 1224$

Endereço para Correspondência

Dra. Lisieux Eyer de Jesus

Rua Presidente Domiciano, 52/801

24210-270 - Niterói-RJ

E-mail: lisieux@openlink.com.br 